\title{
Thermal characterization of Ge-As-Se glasses by differential scanning calorimetry
}

\author{
R. P. Wang $\cdot$ C. J. Zha $\cdot$ A. V. Rode $\cdot$ S. J. Madden $\cdot$ \\ B. Luther-Davies
}

Published online: 27 March 2007

(C) Springer Science+Business Media, LLC 2007

\begin{abstract}
Differential scanning calorimetry (DSC) was performed at different heating rates under non-isothermal conditions to probe the glass transition kinetics of several Ge-As-Se glasses with different chemical compositions. The change of the glass transition temperature $T_{\mathrm{g}}$ with the heating rate and composition was investigated and the activation energy of the glass transition was estimated. The fragility index was calculated and the correlation to the mean coordination number of $\mathrm{Ge}-\mathrm{As}-\mathrm{Se}$ glasses was discussed.
\end{abstract}

\section{Introduction}

Chalcogenide glasses, typically non-oxide based glasses containing the chalcogen elements $\mathrm{S}, \mathrm{Se}$ and $\mathrm{Te}$, have received much attention for many applications. They exhibit many attractive optical properties including high refractive index, excellent transmission at $1.55 \mu \mathrm{m}$, large optical nonlinearity, ultrafast broad-band response, and low optical loss [1-3]. The low glass transition temperature $T_{\mathrm{g}}$ of the amorphous state of these twofold coordinated pure chalcogen elements can be increased by adding three-fold As and four-fold Ge coordinated elements [4]. Assuming Ge, As and Se to possess a coordination number of 4, 3 and 2 respectively, mean coordination number $(\mathrm{MCN})$, which is

R. P. Wang $(\bowtie) \cdot$ C. J. Zha · A. V. Rode .

S. J. Madden - B. Luther-Davies

Centre for Ultrahigh Bandwidth Device for Optical System (CUDOS), Laser Physics Centre, Research School of Physical Science and Engineering, Australian National University, Canberra ACT 0200, Australia

e-mail: rpw111@rsphysse.anu.edu.au defined as a sum of their respective concentration times their coordination number, can be achieved [5]. For binary and ternary chalcogenide glasses, many physical properties have been found to correlate with the MCN. When the MCN increases to 2.4, Phillips and Thorpe [6, 7] predicted a floppy-to-rigid transition in the glass network which has been confirmed by Raman spectroscopy measurements [8]. Boolchand found an intermediate phase bound by the two transitions, revealing that the glass transition becomes almost thermally reversing in this composition window. This floppy-intermediate-stressed rigid phase transition is classified in terms of a structure-based approach to the elastic thresholds. Defining $n_{\mathrm{c}}$ as the count of Lagrangian bonding constraints per atom, intermediate phase consists of optimally constrained $\left(n_{\mathrm{c}}=3\right)$ backbones that appear between mechanically floppy (under constrained, $n_{\mathrm{c}}<3$ ) and stressed rigid (over constrained, $n_{\mathrm{c}}>3$ ) phases [5-7]. The intermediate phase with a thermally reversing window is closely related to the ageing behavior, and thus is important for the application of the glasses.

In Ge-As-Se ternaries containing equal concentrations of As and Ge, nucleation of binary nanocrystalline phases is suppressed due to the same concentration of $\mathrm{As}\left(\mathrm{Se}_{1 / 2}\right)_{3}$ pyramids and $\mathrm{Ge}\left(\mathrm{Se}_{1 / 2}\right)_{4}$ tetrahedra [5, 8-11]. Therefore the so-called nanophase separation which is characteristic of the global maximum in $T_{\mathrm{g}}$ on a composition dependence plot, cannot be observed in $\mathrm{Ge}_{x} \mathrm{As}_{x} \mathrm{Se}_{1-2 x}$ system. However, a global maximum of $T_{\mathrm{g}}$ near $\mathrm{MCN}=2.8$ was found in ternary $\left(\mathrm{Ge}_{2} \mathrm{Se}_{3}\right)_{x}\left(\mathrm{As}_{2} \mathrm{Se}_{3}\right)_{1-x}$ glasses [12]. To resolve this difference, in this paper we investigated the glass transition kinetics of Ge-As-Se glasses with five different chemical compositions, $\mathrm{Ge}_{33} \mathrm{As}_{12} \mathrm{Se}_{55}, \mathrm{Ge}_{22} \mathrm{As}_{20} \mathrm{Se}_{58}, \mathrm{Ge}_{11} \mathrm{As}_{22} \mathrm{Se}_{67}$, $\mathrm{Ge}_{5} \mathrm{As}_{38} \mathrm{Se}_{57}$ and $\mathrm{Ge}_{5} \mathrm{As}_{30} \mathrm{Se}_{65}$. The activation energy of the thermal relaxation processes present in the glasses was also studied. The concentration of $\mathrm{As}\left(\mathrm{Se}_{1 / 2}\right)_{3}$ pyramids and 
$\mathrm{Ge}\left(\mathrm{Se}_{1 / 2}\right)_{4}$ tetrahedra would not be equal in these five $\mathrm{Ge}-$ As-Se glasses. Therefore the investigation may provide further information regarding nanophase separation and whether the Boolchand's intermediate phase still exists since these five samples span a MCN from 2.4.to 2.8 which is characteristic of stressed-rigid glasses. Our preliminary results show a linear correlation between logistic $T_{\mathrm{g}}$ and $\mathrm{MCN}$. By changing the heating rate, the activation energy of the glass transition was derived and the fragility index was calculated for each glass. The dependence of these two parameters on MCN seems to support the existence of a thermally reversing window. The possible physical origin of these results is also discussed.

\section{Experiments}

High purity germanium, arsenic and selenium metals were used as starting materials. They were weighed within a dry nitrogen-filled glove box, and then introduced into a pre-baked quartz ampoule before being sealed under vacuum. The sealed ampoule was placed into a rocking furnace, which was heated to $900{ }^{\circ} \mathrm{C}$ at a ramp rate of $3{ }^{\circ} \mathrm{C} / \mathrm{min}$. To obtain good homogeneity of the melt, the ampoule was rocked for $2 \mathrm{~h}$ and the melt liquid was then held statically in the furnace for $24 \mathrm{~h}$. After the melt was removed from the furnace and quenched in air, the ampoule was subsequently annealed in another furnace for $15 \mathrm{~h}$ at a temperature slightly below $T_{\mathrm{g}}$ in order to reduce the internal stress. A detailed description of the sample preparation can be seen in our previous paper [13]. The glasses obtained were ground into fine powder for differential scanning calorimetry (DSC, Shimadzu DSC-50) measurement. Usually 15-20 mg powder was sealed into Al sample pans and then heated at constant heating rate, and the changes in heat flow with respect to an empty reference pan were measured. No devitrification or crystallization was observed for the samples in this study.

\section{Results and discussion}

The measured $T_{\mathrm{g}}$ of our five samples as a function of MCN, as well as the $T_{\mathrm{g}}$ of those samples with smaller MCN from [9] are shown in Fig. 1. The MCNs are evaluated using the standard procedure described by Tanaka, where the coordination numbers for Ge, As, and Se are 4, 3 and 2, respectively [11]. Thus for the glassy system $\mathrm{Ge}_{\mathrm{a}} \mathrm{As}_{\mathrm{b}} \mathrm{Se}_{\mathrm{c}}(\mathrm{a}+\mathrm{b}+\mathrm{c}=1)$, the value of $\mathrm{MCN}$ is given by:

$\mathrm{MCN}=4 \mathrm{a}+3 \mathrm{~b}+2 \mathrm{c}$,

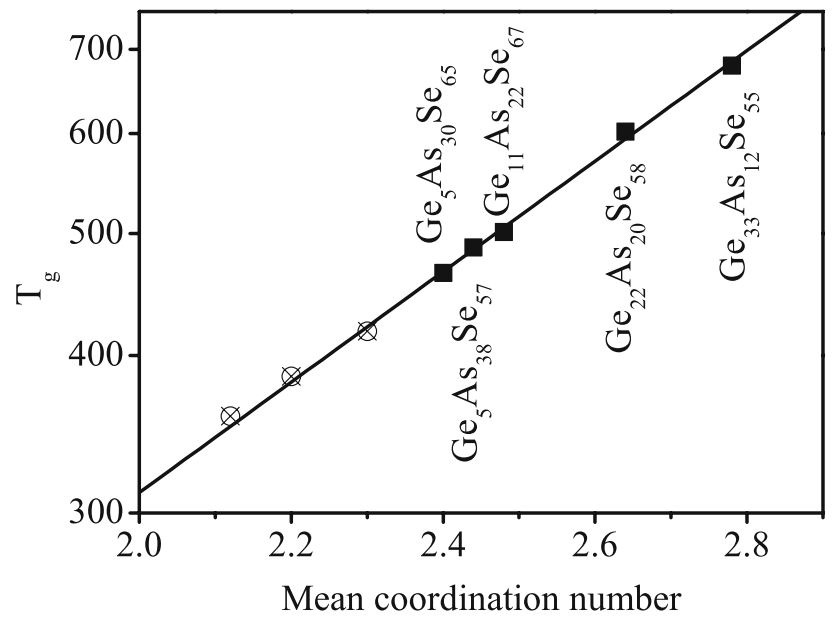

Fig. $1 T_{\mathrm{g}}$ as a function of mean coordination number. The squares represent our five samples, and open circles are those from [9]

As previously mentioned, it is well established that $T_{\mathrm{g}}$ depends on the connectivity, and consequently on the rigidity of the vitreous network $[4,5]$. Therefore adding an element with coordination number larger than two to a linear polymeric structure such as that exhibited by amorphous Se will result in a vitreous matrix with higher $T_{\mathrm{g}}$ proportional to the content of the added atom. In fact a linear correlation between logistic $T_{\mathrm{g}}$ and $\mathrm{MCN}$ has been proposed in chalcogenide glasses [14]. In this study absence of a global maximum in $T_{\mathrm{g}}$ indicates that there is no nanophase separation despite the different concentrations of $\mathrm{As}\left(\mathrm{Se}_{1 / 2}\right)_{3}$ pyramids and $\mathrm{Ge}\left(\mathrm{Se}_{1 / 2}\right)_{4}$ tetrahedra in these materials.

Figure 2 shows DSC curves of $\mathrm{Ge}_{22} \mathrm{As}_{20} \mathrm{Se}_{58}$ samples with different heating rates, where $T_{\mathrm{g}}$ is simply defined as the temperature of the endothermic peak observed during heating. Clearly with increasing heating rate, a more intense endothermic relaxation peak can be observed. Based

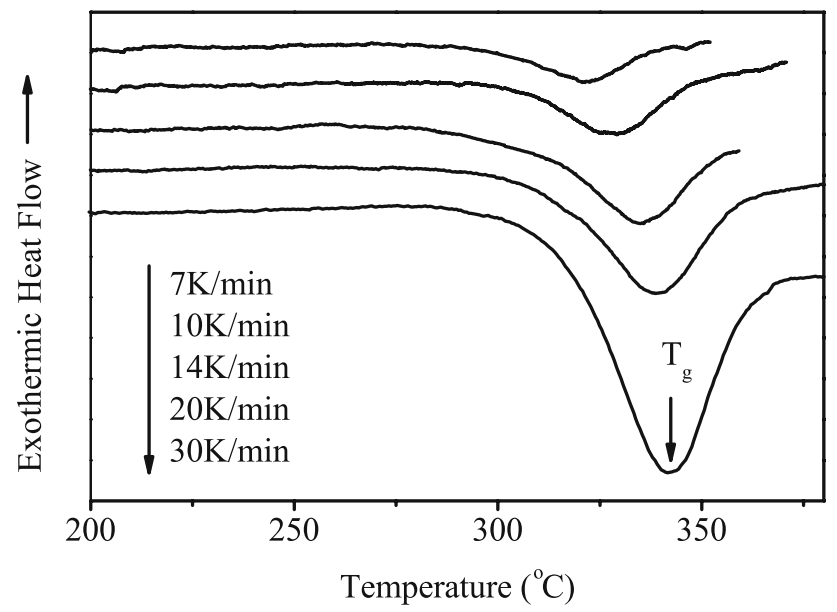

Fig. $2 T_{\mathrm{g}}$ as a function of heating rate 
on Ritland equation, $T_{\mathrm{g}}$ and the heating rate obey the relationship [15],

$d(\ln (\beta)) / d\left(1 / T_{\mathrm{g}}\right)=-\Delta H / R$,

where $\beta, \Delta H$ and $R$ are the heating rate, the apparent activation energy and the universal gas constant, respectively. The plots of $\ln \beta$ versus $1 / T_{\mathrm{g}}$ are shown in Fig. 3 for the five samples investigated in this paper. The apparent activation energy involved in the molecular motions and rearrangements around $T_{\mathrm{g}}$ can be extracted from the slope of each plot, and the results are shown in Fig. 4. It should be emphasized that $\Delta H$ is an apparent activation energy which normally depends on the thermal history and is generally smaller than the "true" glass transition activation energy obtained from cooling scans [16]. (The apparent activation energy depends on the product of partition parameter $x$ and the actual activation energy as observed in cooling scans). Nonetheless, we can still use $\Delta H$ to examine how it changes with the composition and $\mathrm{MCN}$.

The fragility indexes of these five samples were calculated using the relation of $F=\Delta H /\left(T_{g} \ln (10)\right)$ in which $\Delta H$ is determined from heating scans vis-à-vis cooling scans expressed in units of temperature and $T_{\mathrm{g}}$ is glass transition temperature [17]. The calculated values are slightly lower than those obtained by viscoelastic shear compliance and modulus measurements in the tensile modes for $\mathrm{Ge}_{10} \mathrm{As}_{10} \mathrm{Se}_{80}$ and $\mathrm{Ge}_{13} \mathrm{As}_{13} \mathrm{Se}_{74}$ [17].

Since the works of Phillips [6] and Tanaka [11], it is well accepted that glassy covalent networks exhibit two topological thresholds. Describing these glassy structures by means of virtual atoms bonded together with a $\mathrm{MCN}$,

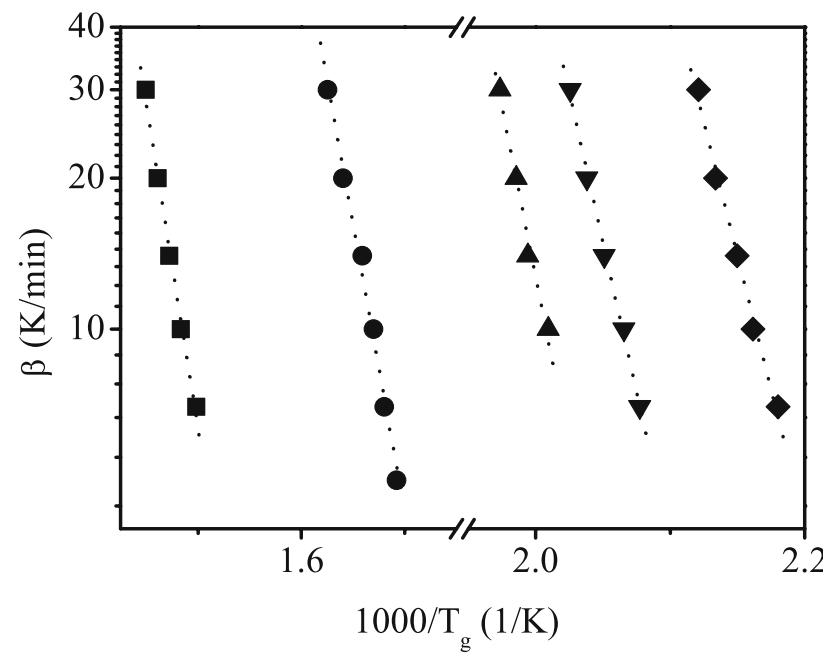

Fig. 3 Plots of $T_{\mathrm{g}}$ versus $\ln \beta$ for the five samples, $\mathrm{Ge}_{33} \mathrm{As}_{12} \mathrm{Se}_{55}(\boldsymbol{\varpi})$, $\mathrm{Ge}_{22} \mathrm{As}_{20} \mathrm{Se}_{58}(\bullet), \mathrm{Ge}_{11} \mathrm{As}_{22} \mathrm{Se}_{67}(\boldsymbol{\Delta}), \mathrm{Ge}_{5} \mathrm{As}_{38} \mathrm{Se}_{57}(\boldsymbol{\nabla})$ and $\mathrm{Ge}_{5} \mathrm{As}_{30} \mathrm{Se}_{65}$

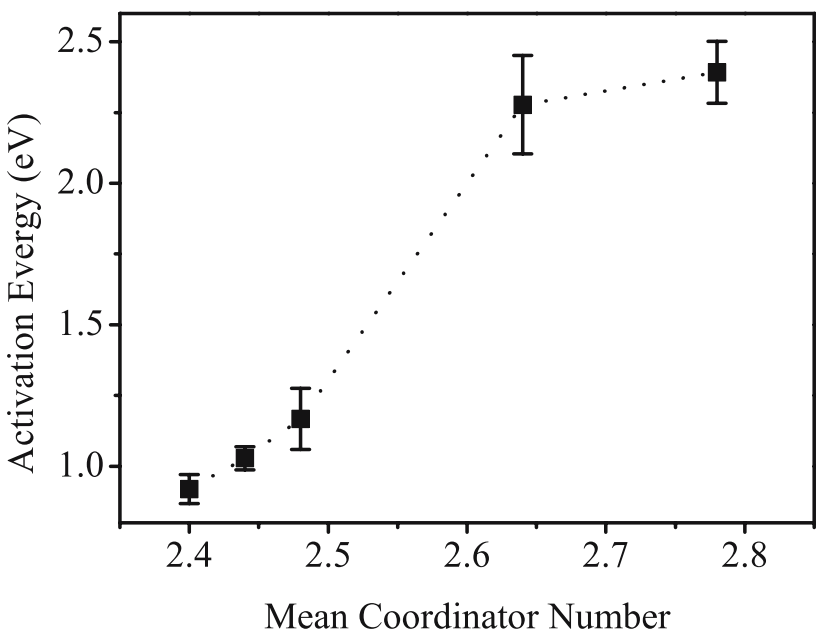

Fig. 4 Activation energy as a function of mean coordination number

these two thresholds appear at MCNs of 2.4 and 2.67, respectively, corresponding to the chemical composition dependence of floppy-intermediate-stressed rigid phase transition. On the other hand, instead of rigid transition involving a change in network dimensionality [11], nanoscale phase separation [12] was proposed to explain the network anomalies near $\mathrm{MCN}=2.6$ in $\left(\mathrm{Ge}_{2} \mathrm{~S}_{3}\right)_{x}\left(\mathrm{As}_{2} \mathrm{~S}_{3}\right)_{1-x}$ systems. Absence of the global maximum of $T_{\mathrm{g}}$ in these preliminary results seems to rule out the possibility of nanoscale phase separation in our $\mathrm{Ge}-\mathrm{As}-\mathrm{Se}$ systems. A detailed investigation of the compositional dependence of $T_{\mathrm{g}}$ will be helpful to elucidate this point.

We note that a prerequisite condition to get the global maximum of $T_{\mathrm{g}}$ is the existence of $\mathrm{As}_{4} \mathrm{Se}_{4}$ monomers which can lead to a progressively loss of the $\operatorname{As}\left(\operatorname{Se}_{1 / 2}\right)_{3}$ backbone and therefore a loss of global connectivity of network. For Ge-As-Se ternary glasses, the electronegativity difference between $\mathrm{Ge}(2.01)$ and $\mathrm{Se}(2.48)$ is larger than that between $\operatorname{As}(2.18)$ and $\operatorname{Se}(2.48)$ [18]. This suggests that Se will first select to covalently bond with $\mathrm{Ge}$ rather than As atoms to low free energy of all network structure. For the Se-rich glasses like $\mathrm{Ge}_{11} \mathrm{As}_{22} \mathrm{Se}_{67}$ and $\mathrm{Ge}_{5} \mathrm{As}_{30} \mathrm{Se}_{65}$, Se chains could be part of the backbone of the network except the tetrahedral $\mathrm{Ge}\left(\mathrm{Se}_{1 / 2}\right)_{4}$ and pyramidal $\left(\mathrm{AsSe}_{3 / 2}\right)_{2}$ units. Moreover, optimally coordinated quasi-tetrahedal Se- $\mathrm{As}\left(\mathrm{Se}_{1 / 2}\right)_{3}$ units could also be formed in Se-rich ternary glasses [9]. On the other hand, for the Se-poor glasses like $\mathrm{Ge}_{33} \mathrm{As}_{12} \mathrm{Se}_{55}$ and $\mathrm{Ge}_{22} \mathrm{As}_{20} \mathrm{Se}_{58}$, $\mathrm{Ge}-\mathrm{Ge}$ and As-As bonds can corporate into the backbone to suppress the formation of binary nanocrystal phase. It has also been found that in $\mathrm{As}_{x} \mathrm{Se}_{1-x}$ binary, ethylene-like $\mathrm{As}_{2} \mathrm{Se}\left({ }_{1 / 2}\right)_{4}$ units were firstly formed as a part of the network, and then gradually transformed into $\mathrm{As}_{4} \mathrm{Se}_{4}$ monomers at $x>0.5$ [19]. If it is true for Se-poor ternary $\mathrm{Ge}-\mathrm{As}-\mathrm{Se}$ glasses, instead of forming $\mathrm{As}_{4} \mathrm{Se}_{4}$ monomers, As atoms will share Se atoms which have a priority to bond 


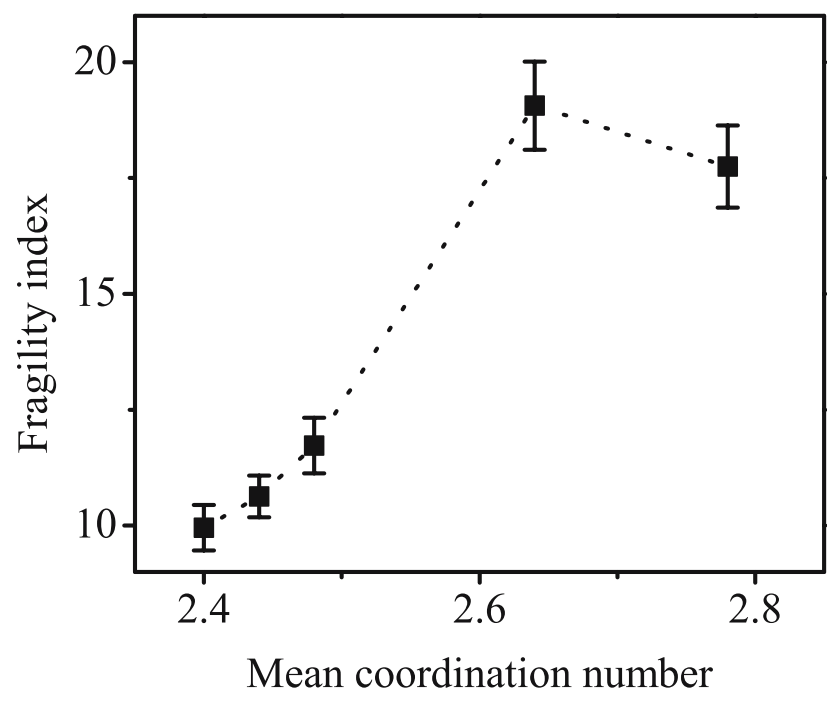

Fig. 5 Fragility index as a function of mean coordination number

with Ge atoms because of the difference of the electronegativity. Therefore we conclude that for both Se-rich and -poor ternary glasses, the independent $\mathrm{As}_{4} \mathrm{Se}_{4}$ monomers are hardly formed. That is the reason why we cannot observe the global maximum of $T_{\mathrm{g}}$.

Noteworthy is a discontinuous change of activation energy and fragility index at the MCN around 2.6 in Figs. 4 and 5 , which is in good agreement with the rigidity transition around 2.67 predicted by the mean field theory. The MCN dependence of decreasing tendency of activation energy and fragility index is in qualitative agreement with those in [9], supporting the existence of Boolchand's intermediate phase in the glassy system.

\section{Conclusion}

In summary, DSC measurements were performed to investigate five Ge-As-Se glassy samples with different compositions. A linear correlation between logistic $T_{\mathrm{g}}$ and could be explained by the formation tendency of the different structural units in the glassy backbones. The change of the glass transition temperature $T_{\mathrm{g}}$ with the heating rate and composition was investigated. The activation energy of the glass transition was derived and the fragility index was calculated. Their correlation to the MCN of $\mathrm{Ge}-\mathrm{As}-\mathrm{Se}$ glasses was discussed.

Acknowledgment This research was supported by Australian Research Council through its Centre of Excellence, Discovery and Federation Fellow Programs.

\section{References}

1. A. Schulte, C. Rivero, K. Richardson et al. Opt. Commun. 198, 125 (2001)

2. A.C. Van Popta, R.G. DeCorby, C.J. Haugen, T. Robinson, J.N. McMullin, Opt. Express 10, 639 (2002)

3. V.Q. Nguyen, J.S. Sanghera, J.A. Freitas, I.D. Aggarwal, I.K. Lloyd, J. Non-Cryst. Solids 248, 103 (1999)

4. M.A. PoPescu, Non-crystalline Chalcogenides (Kluwer Academic Publishers, 2001)

5. P. Boolchand, C.R. Chimie 5, 713 (2002)

6. J.C. Phillips, J. Non-Cryst. Solids 34, 153 (1979)

7. M.F. Thorpe, J. Non-Cryst. Solids 57, 355 (1983)

8. D. Selvanathan, W.J. Bresser, P. Boolchand, Phys. Rev. B 61, 15061 (2000)

9. Y. Wang, P. Boolchand, M. Micoulant, Europhys. Lett. 52, 633 (2000)

10. R. Bohmer, C.A. Angell, Phys. Rev. B 45, 10091 (1992)

11. K. Tanaka, Phy. Rev. B 39, 1270 (1989)

12. S. Mamedov, D.G. Georgiev, T. Qu, P. Boolchand, J. Phys. Condens. Matter 15, 2397 (2003)

13. C.J. Zha, B.L. Davies, R.P. Wang, A. Smith, A. Prasad, R.A. Jarvis, S. Madden, A.Rode, in Proceedings of ACOFT 2006, Melbourne, Australia, 10-13 July 2006

14. R. Kerner, M. Micoulaut, J. Non-Cryst. Solids 210, 298 (1997)

15. H. N. Ritland, J. Am. Ceram. Soc. 37, 370 (1954)

16. S.O. Kasap, S. Yannacopoulos, Phys. Chem. Glasses 31, 71 (1990)

17. R. Bohmer, K.L. Ngai, C.A. Angell, D.J. Plazek, J. Chem. Phys. 99, 4201 (1993)

18. See http://www.webelements.com/webelements

19. B. Effey, R.L. Cappelletti, Phys. Rev. B.59, 4119 (1999) 